\title{
Leucopenia resulting from a drug interaction between azathioprine or 6-mercaptopurine and mesalamine, sulphasalazine, or balsalazide
}

\author{
P W Lowry, C L Franklin, A L Weaver, C L Szumlanski, D C Mays, E V Loftus,
} W J Tremaine, J J Lipsky, R M Weinshilboum, W J Sandborn

\begin{abstract}
Aim-We evaluated the effect of coadministration of sulphasalazine, mesalamine, and balsalazide on the pharmacokinetics and pharmacodynamics of azathioprine and 6-mercaptopurine.

Methods-Thirty four patients with Crohn's disease receiving azathioprine or 6-mercaptopurine were enrolled in an eight week non-randomised parallel group drug interaction study and treated with mesalamine 4 g/day, sulphasalazine 4 g/day, or balsalazide $6.75 \mathrm{~g} / \mathrm{day}$. The primary outcome measure was the occurrence of clinically important leucopenia during the study, defined separately as total leucocyte counts $<3.0 \times 10^{9} / 1$ and $\leqslant 3.5 \times 10^{9} / 1$. Whole blood 6-thioguanine nucleotide concentrations were determined. Results-Three patients could not be evaluated for the primary outcome measure. In the remaining 31 patients, the frequency of total leucocyte counts $<3.0$ and $\leqslant 3.5$ were: $1 / 10$ and $5 / 10$ in the mesalamine group; $1 / 11$ and $6 / 11$ in the sulphasalazine group; and $0 / 10$ and $2 / 10$ in the balsalazide group. There were significant increases in mean whole blood 6-thioguanine nucleotide concentrations from baseline at most time points in the mesalamine and sulphasalazine groups but not in the balsalazide group.

Conclusions-In patients with Crohn's disease receiving azathioprine or 6-mercaptopurine, coadministration of mesalamine, sulphasalazine, and possibly balsalazide results in an increase in whole blood 6-thioguanine nucleotide concentrations and a high frequency of leucopenia.

(Gut 2001;49:656-664)
\end{abstract}

Keywords: azathioprine; 6-mercaptopurine; inflammatory bowel disease; leucopenia; mesalamine; sulphasalazine; balsalazide

The thiopurine 6-mercaptopurine (6-MP) and its prodrug azathioprine (AZA) are effective therapies for inducing and maintaining remission in patients with Crohn's disease (CD). ${ }^{1}$ Thiopurines are metabolised by the enzyme thiopurine methyltransferase (TPMT) to form the metabolite 6-methylmercaptopurine (6MMP). ${ }^{2}$ The level of TPMT activity (phenotype) in red blood cells (RBCs) and other tissues is controlled by a common genetic polymorphism. ${ }^{3}$ The gene for TPMT is located on the short arm of chromosome $6,{ }^{4}$ and to date eight variant alleles (genotypes) have been reported to decrease TPMT activity. ${ }^{5}$ These polymorphisms result in a trimodal distribution of TPMT activity; in the general population with low activity $(<5.0 \mathrm{U} / \mathrm{ml}$ RBCs) occurring at a frequency of $0.3 \%$; intermediate activity (5.0-13.7 U/ml RBCs) occurring at a frequency of $11.1 \%$; and normal or high activity $(13.8-25.1 \mathrm{U} / \mathrm{ml} \mathrm{RBCs})$ occurring at a frequency of $88.6 \% .^{3}$ Patients with low or intermediate TPMT activity phenotypes who are treated with standard doses of AZA or 6-MP are at risk of myelosuppression caused by excess accumulation of the active thiopurine metabolite 6-thioguanine nucleotide (6TGN).$^{67}$

Benzoic acid derivatives such as mesalamine (5-aminosalicylic acid (5-ASA)), N-acetyl-5ASA (the major 5-ASA metabolite), and 5-ASA prodrugs such as sulphasalazine (5ASA linked to sulphapyridine by a diazo bond), olsalazine (two molecules of 5-ASA linked by a diazo bond), and balsalazide (5-ASA linked to an inert carrier molecule 4-aminobenzoyl-balanine by a diazo bond) have been reported to reversibly inhibit the activity of TPMT in vitro. ${ }^{8}$ These medications are widely used in the treatment of CD, and it is common practice to continue their administration when therapy with AZA or 6-MP is initiated in refractory patients. A case report has suggested that coadministration of 6-MP and olsalazine in a patient with CD who had a TPMT activity at the lower end of the normal range led to bone marrow suppression. ${ }^{10}$ Based on these observations, we conducted an eight week nonrandomised parallel group drug interaction study of mesalamine $4 \mathrm{~g}$ /day, sulphasalazine $4 \mathrm{~g}$ /day, or balsalazide $6.75 \mathrm{~g} /$ day in patients with CD who were receiving AZA or 6-MP.

\section{Methods}

SELECTION OF PATIENTS

The study was performed between December 1998 and September 1999 at the Mayo Clinic in Rochester, MN. Potential patients were identified by cross referencing a Mayo Clinical Laboratory database of all patients who had

Abbreviations used in this paper: 6-MP,

6-mercaptopurine; AZA, azathioprine; CD, Crohn's disease; TPMT, thiopurine methyltransferase; 6-MMP, 6-methylmercaptopurine; RBC, red blood cell;

6-TGN, 6-thioguanine nucleotides; 5-ASA, 5-aminosalicylic acid; WBC, white blood cell; IBDQ, inflammatory bowel disease questionnaire; IBD, inflammatory bowel disease. 
RBC TPMT activity measured between 1992 and June 1998 with an institutional database of all patients with inflammatory bowel disease (IBD) evaluated at Mayo Clinic Rochester (identified by ICD9 code diagnoses for CD and ulcerative colitis) during that same time period. The medical records of these patients were reviewed for eligibility criteria. Eligible patients were: at least 18 years of age; had CD; had been treated for a minimum of 16 weeks with either AZA or 6-MP, with a stable dose for at least eight weeks; and had a white blood cell (WBC) count $\geqslant 3.0 \times 10^{9} / 1$. Patients receiving stable doses of antibiotics or prednisone at a dose $\leqslant 20 \mathrm{mg} /$ day or rectal steroids for at least two weeks, and patients receiving a stable regimen of infliximab every eight weeks for at least 16 weeks were eligible.

Patients receiving prednisone $>20 \mathrm{mg} /$ day were not eligible, nor were those who had received mesalamine, sulphasalazine, olsalazine, balsalazide, methotrexate, or mycophenolate mofetil within two months, or cyclosporin, tacrolimus, or investigational biological agents within three months. In addition, the following patients were not eligible: pregnant or breast feeding women, those requiring immediate surgery, those with a septic complication or abscess, and those with known intolerance to mesalamine or balsalazide (patients with intolerance to sulphasalazine were eligible but were treated with mesalamine or balsalazide). $\mathrm{Pa}-$ tients with low RBC TPMT activity $(<5.0$ $\mathrm{U} / \mathrm{ml} \mathrm{RBCs})^{3}$ were excluded as were those with a planned hospitalisation within eight weeks, those with recent alcohol or drug abuse, those with clinically significant renal or hepatic disease, and those who had received allopurinol within one week. Patients were recruited in person or by introductory letter or phone call in conjunction with recruitment for another study. ${ }^{11}$ The institutional review board of the Mayo Clinic approved the study, and all participants gave written informed consent.

STUDY MEDICATIONS

The mesalamine formulation used was Pentasa capsules (Hoechst Marion Rousel, Kansas City, Missouri, USA) that release mesalamine continuously from the duodenum to the rectum. Patients received four $250 \mathrm{mg}$ capsules of Pentasa orally four times daily. The sulphasalazine formulation used was Azulfidine tablets (Pharmacia Upjohn, Kalamazoo, Michigan, USA) that dissolve in the stomach and duodenum. Patients received two $500 \mathrm{mg}$ tablets of Azulfidine orally twice daily and then increased the daily dose by two $500 \mathrm{mg}$ tablets every three days until a dose of two $500 \mathrm{mg}$ tablets four times daily was reached. The balsalazide formulation used was Colazide tablets (Astra Draco, Lund, Sweden) that dissolve in the stomach and duodenum. At the time of the study, balsalazide was an investigational drug in the USA and was obtained from the UK under an investigational drug number (IND) from the Food and Drug Administration. Patients received three $750 \mathrm{mg}$ tablets of Colazide orally three times daily. In addition, patients continued their pre-study doses of azathioprine (either Imuran from Glaxo Wellcome, Research Triangle Park, North Carolina, USA or Faro Pharmaceuticals, Toronto, Ontario, Canada, or generic azathioprine) or 6-mercaptopurine (Purinethol; Glaxo Wellcome) throughout the eight week study.

If patients developed laboratory abnormalities consistent with hepatotoxicity (aspartate aminotransferase $>5 \times$ normal or alkaline phosphatase $>3 \times$ normal), moderate leucopenia (total WBC count $<3.0 \times 10^{9} / 1$, normal range $3.5-10.5 \times 10^{9} / 1$ ), or thrombocytopenia (platelet count $\left.<100 \times 10^{9} / 1\right)$, then mesalamine, sulphasalazine, or balsalazide was discontinued and patients were removed from the study prematurely. Decisions regarding discontinuation or dose adjustments of mesalamine, sulphasalazine, or balsalazide in patients who experienced infection, fever, rash, arthralgias, malaise, heartburn or dyspepsia, nausea, headache, and worsening diarrhea were handled on a case by case basis. If patients developed pancreatitis or malignancy, AZA or 6-MP was discontinued. Compliance with mesalamine, sulphasalazine, or balsalazide was determined by pill count.

\section{DESIGN OF THE STUDY}

The eight week study was a parallel group drug interaction study. We selected a study design in which each subject served as his or her own control because we believed that interpatient variation in the metabolism of AZA and 6-MP would make it difficult to interpret the results of a placebo controlled trial. We did not randomise or blind patients to treatment assignment in order to avoid randomising patients with a history of intolerance to sulphasalazine (up to $30 \%$ of patients) to treatment with sulphasalazine. We considered randomising patients with no history of sulphasalazine intolerance among the three treatment groups and separately randomising patients with a history of sulphasalazine intolerance (estimated to be up to $30 \%$ of patients or more) to treatment with mesalamine or balsalazide. However, we decided against this approach because it would have led to unequal and unpredictable distributions of patients to the mesalamine and balsalazide groups.

At entry, each patient's demographic characteristics, medical history, and current medications were recorded. At the baseline and week 8 visits, and in the event that treatment was discontinued, a physical examination and quality of life assessment were conducted, and patients were asked whether any adverse events had occurred. Blood was obtained for serum chemistries at baseline and at week 8 , and for a complete blood count at baseline and weeks $2,4,6$, and 8.

Quality of life at baseline was assessed with the self administered inflammatory bowel disease questionnaire (IBDQ), a previously validated instrument with four parts (bowel function, emotional status, systemic symptoms, and social function). The total score on this index ranges from 32 to 224, with higher scores indicating better quality of life. The 
scores of patients in remission usually range from 170 to $190 .{ }^{12}$ All adverse events were recorded and graded according to World Health Organization criteria.

At baseline and at weeks 2, 4, 6, and 8 (or at the time of withdrawal or termination from the study) blood was obtained one hour after administration of AZA or 6-MP for measurement of whole blood concentrations of 6-TGN. Similarly, at week 8 (or at the time of withdrawal or termination from the study), blood was obtained one hour after administration of mesalamine, sulphasalazine, or balsalazide for plasma concentrations of 5-ASA, $\mathrm{N}$-acetyl-5-ASA, sulphasalazine, and balsalazide. Blood was also obtained at baseline and week 8 (or at the time of withdrawal or termination of the study) for RBC TPMT activity. Duodenal mucosa was obtained by oesophagogastroduodenoscopy with biopsy of the second portion of the duodenum at baseline and week 8 (or at the time of withdrawal or termination of the study) for mucosal concentrations of 6-TGN. The endoscopic biopsies were placed immediately on dry ice and stored at $-80^{\circ} \mathrm{C}$ until assay. Duodenal mucosal tissue samples were then thawed, washed, blotted dry, weighed, minced, and diluted 1:1 with saline, homogenised, and then assayed. The results are expressed per gram of wet mucosal tissue.

RBC TPMT activity was determined by a radiochemical enzymatic assay method (Mayo Medical Laboratories, Rochester, Minnesota, USA) as previously described. ${ }^{3}$ Total 6-TGN was assayed by high pressure liquid chromatography using the Erdmann method with minor modifications. ${ }^{13}$ Whole blood rather than washed RBCs was used, following a study which reported that the total 6-TGN concentration is not significantly different in whole blood compared with washed RBCs (unpublished data). Total intracellular 6-TGN concentration represents the total RBC 6-TGN content consisting of a composite of the mono-, di-, and triphosphate nucleotides. Mucosal 6-TGN concentrations were measured as previously described using modifications of procedures for other solid organs. ${ }^{14}$ Plasma concentrations of 5-ASA, N-acetyl-5-ASA, sulphasalazine, free sulphapyridine, $\mathrm{N}$ acetyl-sulphapyridine, total sulphapyridine (free sulphapyridine $+N$-acetyl-sulphapyridine), and balsalazide were measured by methods previously described, with minor modifications. ${ }^{15} 16$

OUTCOMES AND STATISTICAL ANALYSIS

Baseline characteristics and adverse events were summarised by treatment subgroup on all patients enrolled in the study. Analysis of primary and secondary outcome measures was based on an intention to treat sample which included all patients who had received at least one dose of a study medication and had at least one evaluation for leucopenia after starting the study medication. The primary outcome measure of the study was the estimated proportion of clinically important leucopenia occurring in each treatment group at any time during the trial, defined separately as total WBC counts
$<3.0 \times 10^{9} / 1$ and $\leqslant 3.5 \times 10^{9} / 1$. The definition of clinically important leucopenia as a total WBC count of $<3.0 \times 10^{9} / 1$ was specified prior to the study. A second definition of clinically important leucopenia as a total WBC count of $\leqslant 3.5 \times 10^{9} / 1$ (which aligns more closely with the WBC count that would lead clinicians to alter the AZA or 6-MP dose) was specified after completion of the study. Secondary outcome measures were: (1) change from baseline in total WBC counts; (2) changes from baseline in 6-TGN concentrations in whole blood and duodenal mucosa; and (3) change from baseline in RBC TPMT activity to assess the possibility of enzyme "induction".

Estimates of the proportions of clinically important leucopenia were computed at baseline and at 2, 4, 6, and 8 weeks of treatment, and for the total eight week study, for each study subgroup. Ninety five per cent confidence intervals (95\% CIs) for the proportions were based on exact binomial probabilities. Evaluation of the proportion with clinically important leucopenia defined as total leucocyte counts $<3.0 \times 10^{9} / 1$ was made based on a one sided one sample $t$ test for proportions (see sample size below). Comparison of the proportion with clinically important leucopenia defined as total leucocyte $\leqslant 3.5 \times 10^{9} / 1$ observed at baseline and over the eight week study was made based on exact binomial theory using a two sided McNemar's test for correlated proportions. For each secondary outcome measure, the $\mathrm{F}$ test from a one way repeated measures analysis of variance (ANOVA) model, with time as the quantitative variable, was used to test for a linear trend over time within each subgroup. In the presence of a significant $\mathrm{F}$ test, the paired $t$ test was used to compare pretreatment and post-treatment values of the secondary outcome measures within each subgroup. Concentrations less than the lower limit of quantitation were underestimated by assuming a concentration of zero. For patients withdrawing from the study prematurely, assessments obtained at the time of withdrawal were utilised in the analysis of the final assessments. Statistical comparisons were limited to comparisons over time within the subgroups. No formal statistical comparisons between the subgroups were performed as patients were not randomly assigned to the groups. All calculated $p$ values were two sided unless otherwise specified above, and $\mathrm{p}$ values less than 0.05 were considered statistically significant.

\section{SAMPLE SIZE}

Assuming that the proportion of patients with CD developing clinically important leucopenia (defined as a total leucocyte count $<3.0 \times 10^{9} / 1$ ) would be $\leqslant 10 \%$ if there was no effect of combination therapy, there was $80 \%$ power to detect an effect if the true rate was $\geqslant 45 \%$, with $\mathrm{n}=10$. These estimates were based on a one sample test for proportions, using $\sin ^{-1}(p)$ transformation at an $\alpha$ level of 0.05 (one sided). 
Table 1 Baseline characteristics of the patients *

\begin{tabular}{lccc}
\hline Variable & $\begin{array}{l}\text { Mesalamine } \\
\text { group }(n=10)\end{array}$ & $\begin{array}{l}\text { Sulphasalazine } \\
\text { group }(n=12)\end{array}$ & $\begin{array}{l}\text { Balsalazide group } \\
(n=12)\end{array}$ \\
\hline Sex (M/F) & $4 / 6$ & $7 / 5$ & $6 / 6$ \\
Age at entry (y) (mean (SD)) & $39.7(9.7)$ & $36.8(7.8)$ & $38.3(10.7)$ \\
Weight (kg) (mean (SD)) & $66.1(14.9)$ & $76.9(16.5)$ & $84.7(26.0)$ \\
AZA No & 7 & 9 & 8 \\
Dose (mg/kg) (mean (SD)) & $2.0(0.7)$ & $2.0(0.5)$ & $2.1(0.6)$ \\
6-MP No & 3 & 3 & 4 \\
Dose (mg/kg) (mean (SD)) & $1.2(0.3)$ & $1.1(0.2)$ & $1.1(0.3)$ \\
TPMT activity (U/ml RBCs) & & & 18.0 \\
Overall mean & 16.1 & 17.0 & 4.1 \\
Overall SD & 4.2 & 5.6 & $8.3-23.1$ \\
Overall median & 16.9 & 16.0 & 0 \\
Overall range & $8.6-21.2$ & $9.2-28.9$ & $2^{\mathrm{C}}$ \\
No low & 0 & 0 & 10 \\
No intermediate & $2^{\mathrm{A}}$ & $2^{\mathrm{B}}$ & $178(24)$ \\
No normal & 8 & 10 & \\
Inflammatory bowel disease & $186(21)$ & $196(18)$ & \\
questionnaire (mean (SD)) & &
\end{tabular}

$\star 34$ patients were screened, enrolled, and received at least one dose of study medication, and were included in the safety analysis. Elsewhere in the manuscript, results presented are for the intention to treat population (31 patients).

AZA, azathioprine; 6-MP, 6-mercaptopurine; TPMT, thiopurine methyltransferase; RBCs, red blood cells.

For patients with the intermediate TPMT phenotype, TPMT activities in the three treatment groups were: ${ }^{\mathrm{A}}$ mesalamine group 8.6 and $9.3 \mathrm{U} / \mathrm{ml} \mathrm{RBCs}$; ${ }^{\mathrm{B}}$ sulphasalazine group 9.2 and $9.6 \mathrm{U} / \mathrm{ml}$ RBCs; and ${ }^{C}$ balsalazide group 8.3 and $13.6 \mathrm{U} / \mathrm{ml}$ RBCs.

\section{Results}

A total of 951 patients with IBD had TPMT activity measured between 1992 and June 1998. These patients' medical records were reviewed during the fall of 1998 for eligibility criteria for another study. ${ }^{11}$ We identified 376 patients who had either started or continued AZA or 6-MP at the time of their last Mayo Clinic evaluation. These 376 patients were contacted by mail regarding the other study, of whom 217 initially agreed to participate, and 170 actually returned the questionnaires and submitted blood specimens ${ }^{11}$ : 575 patients were ineligible for the other study. ${ }^{11}$ Detailed records of the reasons for ineligibility were not kept but reasons included: patient not started on AZA or 6-MP; patient no longer taking
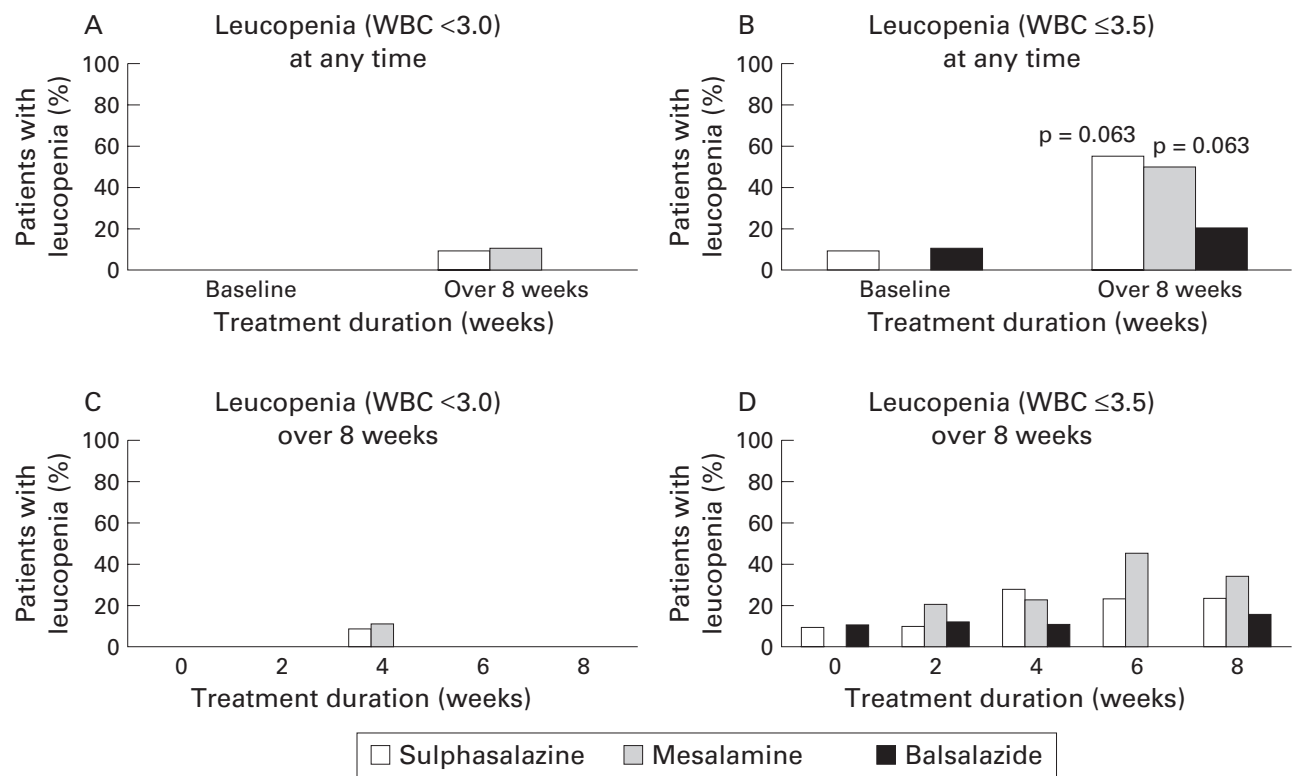

Figure 1 Percentage of patients with Crohn's disease with clinically important leucopenia, according to treatment group. All significant differences between week 0 and subsequent time points for each of the treatment groups are indicated. $(A)$ Clinically important leucopenia (white blood cell $(W B C)$ count $<3.0 \times 10^{9} / l$ ) at any time during the study. (B) Clinically important leucopenia (WBC count $\leqslant 3.5 \times 10^{9} / \mathrm{l}$ ) at any time during the study. The $95 \%$ confidence intervals for this end point for the mesalamine, sulphasalazine, and balsalazide groups are 19-81\%, 23-83\%, and 3-56\%, respectively. (C) Clinically important leucopenia (WBC count $<3.0 \times 10^{9} / \mathrm{ll}$ ) over eight weeks. (D) Clinically important leucopenia (WBC count $\left.\leqslant 3.5 \times 10^{9} / l\right)$ over eight weeks.

AZA or 6-MP because of failure to respond; AZA or 6-MP discontinued after operation; patient discontinued AZA or 6-MP because of toxicity; and other miscellaneous reasons. Of the 170 patients in the other study, 61 were receiving mesalamine, sulphasalazine, or olsalazine and were thus ineligible for the current study. Of the remaining 109 patients, 34 also agreed to participate in the current study and were screened and enrolled. Of those, all 34 patients received at least one dose of study medication and were included in the safety analysis; 10 received mesalamine, 12 received sulphasalazine, and 12 received balsalazide. One patient in the sulphasalazine group and two in the balsalazide group did not have at least one evaluation for leucopenia after receiving the study medication. Thus the intention to treat sample consisted of 31 patients. Baseline characteristics of the three groups of patients are shown in table 1 . Because this was not a randomised trial, formal comparisons of the baseline characteristics for the three treatment groups were not performed. Nevertheless, the groups appeared to be similar except for body weight; differences in body weight may not be relevant because AZA and 6-MP doses (which are based on body weight) are similar. Nine of 10 patients $(90 \%)$ in the mesalamine group, $9 / 12(75 \%)$ in the sulphasalazine group, and $8 / 12(67 \%)$ in the balsalazide group completed the eight week study. Leucopenia at week 4 led to withdrawal at week 6 of one patient $(10 \%)$ in the mesalamine group and one patient $(8.3 \%)$ in the sulphasalazine group. One patient $(8.3 \%)$ in the sulphasalazine group and four $(33 \%)$ in the balsalazide group withdrew due to drug intolerance (headache, abdominal discomfort, diarrhoea). One patient $(8.3 \%)$ in the sulphasalazine group developed melena at

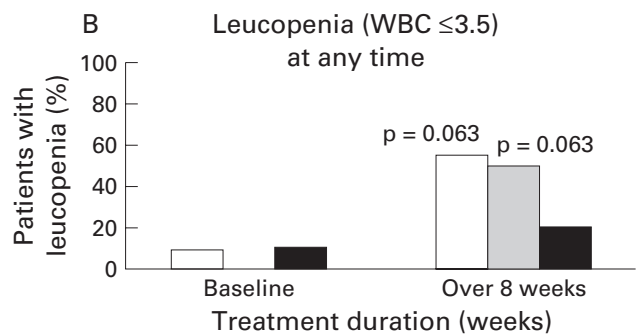




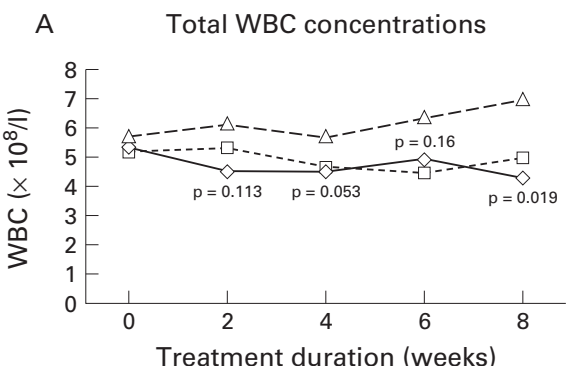

C Duodenal mucosal 6-TGN concentrations

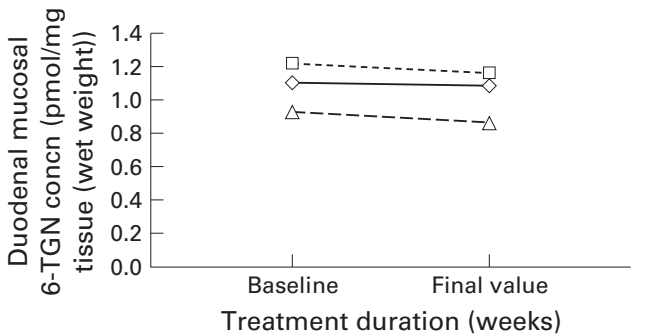

B Whole blood 6-TGN concentrations

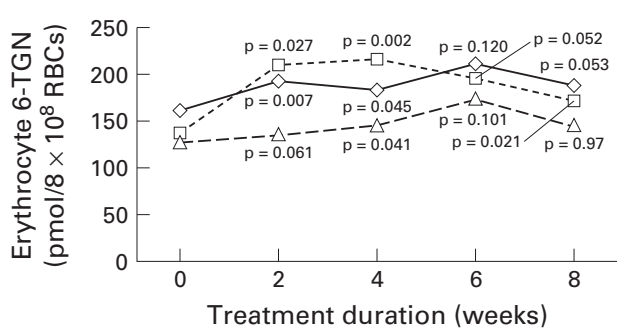

D TPMT activity over 8 weeks

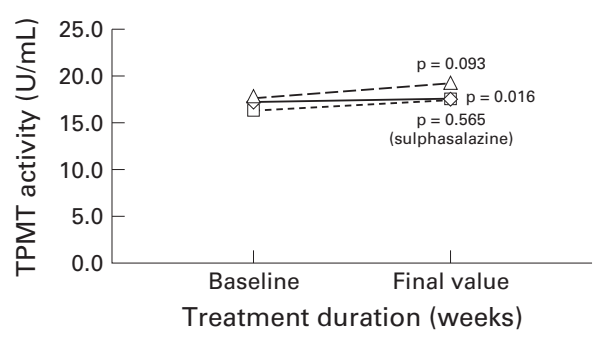

$\diamond$ Sulphasalazine

$\square$ Mesalamine

$\triangle$ Balsalazide

Figure 2 Mean values at each study visit according to treatment group. Both significant and non-significant differences between week 0 and subsequent time points for each of the treatment groups are indicated. (A) Total white blood cell (WBC) concentrations. (B) Whole blood 6-thioguanine nucleotide (6-TGN) concentrations. Mean (95\% confidence intervals) change from baseline for whole blood 6-TGN (pmol/8 $\times 10^{8}$ RBCs) in the mesalamine group at weeks $2,4,6$, and 8 were: 74 (11-136), 71 (36-106), 55 (-1-110), and 43 (8-77). Mean (95\% confidence intervals) change from baseline

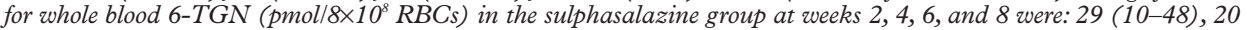

(1-39), 30 (-10-70), and 38 (-1-77). Mean (95\% confidence intervals) change from baseline for whole blood 6-TGN

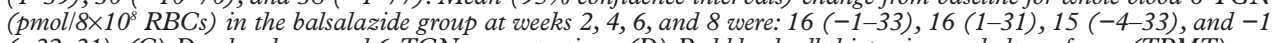
(-32-31). (C) Duodenal mucosal 6-TGN concentrations. (D) Red blood cell thiopurine methyltransferase (TPMT) activity.

week 4 without an obvious source apparent on oesophagogastroduodenoscopy or colonoscopy, and elected to withdraw early from the study.

CLINICALLY IMPORTANT LEUCOPENIA

The rates of clinically important leucopenia over the eight week study using a cut off of WBC count $<3.0 \times 10^{9} / 1$ were slightly increased compared with baseline for mesalamine at $10 \%$ (95\% CI $0.3-45 \%)$ and sulphasalazine at $9 \%$ (95\% CI $0.2-41 \%)$ but not for balsalazide at $0 \%$ (95\% CI 0-31\%) (fig 1A). These trends were not significant. Using a more sensitive cut off of WBC count $\leqslant 3.5 \times 10^{9} / 1$ there were strong trends towards increased rates of clinically important leucopenia over the eight week study compared with baseline for mesalamine $(50 \%$ $v 0 \% ; \mathrm{p}=0.063)$ and sulphasalazine $(55 \% v$ $9 \% ; \mathrm{p}=0.063)$ but not for balsalazide $(20 \% v$ $10 \%$ ) (see fig $1 \mathrm{~B}$ for graphic representation and the legend for fig $1 \mathrm{~B}$ for $95 \% \mathrm{CIs})$. The rates of clinically important leucopenia occurring in each treatment group at baseline and at weeks $2,4,6$, and 8 using WBC count $<3.0 \times 10^{9} / 1$ as the cut off are shown in fig $1 \mathrm{C}$ and, using WBC count $\leqslant 3.5 \times 10^{9} / 1$ as the cut off, in fig $1 \mathrm{D}$. One patient in the mesalamine group and one patient in the sulphasalazine group exited from the study at six weeks due to leucopenia; their WBC counts were $2.8 \times 10^{9} / 1$ and $2.5 \times 10^{9} / 1$, respectively. Mean baseline WBC counts were similar for the three treatment groups, ranging from 5.0 to $5.7 \times 10^{9} / 1$ (fig $2 \mathrm{~A}$ ). Among patients receiving sulphasalazine, mean WBC counts significantly decreased over time $(p=0.018)$ (fig 2A). There was a trend towards lower mean WBC counts over time for patients receiving mesalamine but not for those receiving balsalazide (fig 2A).

6-TGN CONCENTRATIONS IN WHOLE BLOOD AND DUODENAL MUCOSA

Mean baseline whole blood 6-TGN concentrations for patients in the mesalamine, sulphasalazine, and balsalazide groups were 137, 162 , and $128 \mathrm{pmol} / 8 \times 10^{8} \mathrm{RBCs}$, respectively. In each of the three study groups, whole blood 6-TGN concentrations increased after initiation of the study drug (fig 2B). Among patients taking sulphasalazine, mean whole blood 6-TGN concentrations significantly increased over time $(p=0.013)$. Although there was no significant linear trend over time for patients in the mesalamine group $(p=0.15)$, increases in whole blood 6-TGN concentrations (relative to baseline) were statistically significant for most time points. The maximum average change from baseline in the mesalamine group occurred at week 2 (mean change 74 (95\% CI 11-136) $\left.\mathrm{pmol} / 8 \times 10^{8} \mathrm{RBCs}\right)$ and in the sulphasalazine group at week 8 (mean change 38 (95\% CI -1-77) pmol $/ 8 \times 10^{8} \mathrm{RBCs}$ ) (fig $2 \mathrm{~B}$ ). Mean final (week 8 or time of study termination) whole blood 6-TGN concentration for all 31 patients was $168 \mathrm{pmol} / 8 \times 10^{8} \mathrm{RBCs}$. Three of six patients in the sulphasalazine group who developed clinically important leucopenia (WBC count $\leqslant 3.5 \times 10^{9} / 1$ ) had whole blood 6 -TGN concentrations above $168 \mathrm{pmol} / 8 \times 10^{8}$ RBCs; the other three patients with leucopenia in the sulphasalazine group had elevated 
plasma concentrations of total sulphapyridine (see below). In contrast, only one of five patients in the sulphasalazine group who did not develop clinically important leucopenia had whole blood 6-TGN concentrations above $168 \mathrm{pmol} / 8 \times 10^{8} \mathrm{RBCs}$. Similarly, four of five patients in the mesalamine group and one of two patients in the balsalazide group who developed leucopenia had whole blood 6-TGN concentrations above $168 \mathrm{pmol} / 8 \times 10^{8} \mathrm{RBCs}$ compared with one of five patients in the mesalamine group and one of eight patients in the balsalazide group who did not develop leucopenia.

Mean baseline duodenal mucosal 6-TGN concentrations for patients in the mesalamine, sulphasalazine, and balsalazide groups were $1.21,1.10$, and $0.92 \mathrm{pmol} / \mathrm{mg}$ tissue (wet weight). There was no significant change from baseline in mean duodenal mucosal 6-TGN concentration in any of the three treatment groups (fig 2C).

RBC THIOPURINE METHYLTRANSFERASE ACTIVITY Mean (median, range) baseline RBC TPMT activities for patients in the mesalamine, sulphasalazine, and balsalazide groups were 16.1 (16.9, 8.6-21.2), 17.1 (16.0, 9.2-28.9), and $17.8(18.2,8.3-23.1) \mathrm{U} / \mathrm{ml}$ RBCs, respectively. In the mesalamine and balsalazide groups, mean RBC TPMT activities were increased at week 8 (or time of study withdrawal) compared with baseline, and this difference was significant for mesalamine (mean change 1.5, 95\% CI 0.4-2.7) (fig 2D). There was no change from baseline in mean RBC TPMT activity in the sulphasalazine group (fig 2D).

Two of 11 patients in the mesalamine group had intermediate TPMT activity (8.6 and 9.3 $\mathrm{U} / \mathrm{ml} \mathrm{RBCs}$ ), both were taking somewhat reduced medication doses (6-MP $0.86 \mathrm{mg} / \mathrm{kg}$ and AZA $0.68 \mathrm{mg} / \mathrm{kg}$ ), and neither developed clinically significant leucopenia. Similarly, two of 10 patients in the sulphasalazine group had intermediate TPMT activity $(9.2$ and $9.6 \mathrm{U} / \mathrm{ml}$ RBCs), one took a reduced dose of AZA (1.07 $\mathrm{mg} / \mathrm{kg}$ ) and did not develop leucopenia, while the other took a standard dose of AZA (2.02 $\mathrm{mg} / \mathrm{kg}$ ) and developed leucopenia (WBC count $\left.2.8 \times 10^{9} / 1\right)$. Finally, two patients in the balsalazide group had intermediate TPMT activ-

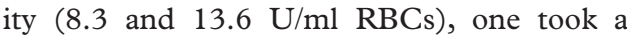

Table 2 Adverse events in the three treatment groups *

\begin{tabular}{llll}
\hline Variable & $\begin{array}{l}\text { Mesalamine } \\
\text { group }(n=10) \\
(n(\%))\end{array}$ & $\begin{array}{l}\text { Sulphasalazine } \\
\text { group }(n=12) \\
(n(\%))\end{array}$ & $\begin{array}{l}\text { Balsalazide } \\
\text { group }(n=12) \\
(n(\%))\end{array}$ \\
\hline No adverse events & 15 & 27 & 14 \\
No patients with adverse events & $7(70)$ & $10(83)$ & $8(67)$ \\
No patients with serious adverse events & $0(0)$ & $1(8)$ & $0(0)$ \\
No patients with severe adverse events & $1(10)$ & $3(25)$ & $4(33)$ \\
$\begin{array}{l}\text { Most frequent adverse events (No events) } \\
\left.\text { Leucopenia (WBC }<3.0 \times 10^{9} / 1\right)\end{array}$ & $1(10)$ & $1(8)$ & $0(0)$ \\
Leucopenia (WBC $\left.\leqslant 3.5 \times 10^{9} / 1\right)$ & $5(50)$ & $6(50)$ & $2(17)$ \\
Headache & $1(10)$ & $6(50)$ & $0(0)$ \\
Nausea & $1(10)$ & $4(33)$ & $1(8)$ \\
Worsening diarrhoea & $1(10)$ & $1(8)$ & $4(33)$ \\
Fatigue & $1(10)$ & $4(33)$ & $1(8)$ \\
\hline
\end{tabular}

$\star 34$ patients were screened, enrolled, and received at least one dose of study medication, and were included in the safety analysis. Elsewhere in the manuscript, results presented are for the intention to treat population (31 patients). standard dose of AZA (2.89 mg/kg) and developed leucopenia (WBC count $3.4 \times 10^{9} / 1$ ), the other took a reduced dose of AZA $(1.15 \mathrm{mg} / \mathrm{kg})$ and also developed leucopenia (WBC count $\left.3.4 \times 10^{9} / 1\right)$.

5-ASA, $N$-ACETYL-5-ASA, SULPHASALAZINE, SULPHAPYRIDINE, $N$-ACETYL-SULPHAPYRIDINE, AND BALSALAZIDE CONCENTRATIONS IN PLASMA The following drug and metabolite concentrations were obtained at week 8 (or at the time of study withdrawal). Mean plasma 5-ASA concentrations for patients in the mesalamine, sulphasalazine, and balsalazide groups were 12.0, 1.2 , and $1.8 \mu \mathrm{M}$, respectively. Mean plasma $\mathrm{N}$-acetyl-5-ASA concentrations for patients in the mesalamine, sulphasalazine, and balsalazide groups were 14.0, 2.6, and $3.2 \mu \mathrm{M}$, respectively. Mean plasma sulphasalazine, free sulphapyridine, $\mathrm{N}$-acetyl-sulphapyridine, and total sulphapyridine concentrations for patients in the sulphasalazine group were 25.4, $49.5,29.3$, and $78.7 \mu \mathrm{M}$, respectively. Mean plasma balsalazide concentration for patients in the balsalazide group was $0.9 \mu \mathrm{M}$. Five of six patients in the sulphasalazine group who developed clinically important leucopenia (WBC count $\leqslant 3.5 \times 10^{9} / 1$ ) had plasma concentrations of total sulphapyridine above the threshold of $50 \mu \mathrm{M}$ that has been reported to be associated with haematological toxicity (see discussion). The other patient with leucopenia had an RBC 6-TGN concentration above 168 $\mathrm{pmol} / 8 \times 10^{8} \mathrm{RBCs}$ (see above).

\section{ADVERSE EVENTS}

The frequency of adverse events occurring during the study in the mesalamine, sulphasalazine, and balsalazide groups are listed in table 2 . In addition to leucopenia which has been discussed above, headache led to early withdrawal of one patient $(8 \%)$ in the sulphasalazine group, and nausea and vomiting in one patient and worsening diarrhoea in three patients led to early withdrawal (33\% combined) in the balsalazide group (these symptoms resolved after balsalazide withdrawal).

\section{Discussion}

We found that coadministration of AZA or 6-MP with mesalamine or sulphasalazine in patients with CD caused an increase in the frequency of clinically important leucopenia (defined as a WBC count $\leqslant 3.5 \times 10^{9} / 1$ ) of $50 \%$ over the eight week study compared with baseline therapy with AZA or 6-MP alone (there was a strong trend towards this difference being statistically significant using the two sided McNemar's test for correlated proportions). The frequency of clinically important leucopenia in patients receiving balsalazide was not significantly different from baseline. Although this definition was specified post hoc, we believe that the magnitude of the effect is so large that it is unlikely to be a statistical artefact. When a more rigorous definition of clinically important leucopenia (WBC count 
$\left.<3.0 \times 10^{9} / 1\right)$ was used as the end point, the frequency of leucopenia was not significantly different from baseline although $10 \%$ of mesalamine treated patients and $8 \%$ of sulphasalazine treated patients required early discontinuation from the study for this reason. In support of these observations, mean WBC count decreased from baseline at most time points tested in patients treated with sulphasalazine. Furthermore, we have reported previously in a cross sectional study of patients receiving AZA or 6-MP that concomitant therapy with mesalamine, sulphasalazine, or olsalazine resulted in a significant decrease in mean total WBC count. ${ }^{11}$ Although we cannot exclude the possibility that leucopenia observed in our study could have occurred in patients treated with AZA or 6-MP alone, the frequencies of leucopenia observed in our study (20-55\%) over an eight week period appear to be substantially greater than rates of $2-11 \%$ reported in clinical trials and large observational studies of AZA and 6-MP therapy for IBD extending over much longer periods of time. ${ }^{17-19}$ Our findings confirm an earlier case report which suggested that the 5-ASA dimer, olsalazine (and possibly other 5-ASA derivatives) may cause leucopenia when coadministered with AZA or 6-MP. ${ }^{10}$ Based on these results, health care providers should be made aware of the increased risk for clinically important leucopenia when co-prescribing mesalamine, sulphasalazine, olsalazine, and possibly balsalazide with AZA or 6-MP.

We also found that coadministration of AZA or 6-MP and mesalamine, sulphasalazine, or balsalazide in patients with CD led to increased mean whole blood 6-TGN concentrations compared with baseline, and that those increases were statistically significant in the mesalamine and sulphasalazine treated patients. Although we cannot exclude the possibility that these increases in mean whole blood 6-TGN concentrations observed in our study could have occurred in patients treated with AZA or 6-MP alone, a previous study suggested no significant variation in mean whole blood 6-TGN concentrations in patients treated with AZA, once steady state concentrations had been achieved after six weeks of therapy. ${ }^{17}$ It is possible that this increase in mean whole blood 6-TGN concentrations is clinically relevant and contributed to the development of leucopenia. Of the 13 patients who developed leucopenia during the study, three of six patients in the sulphasalazine group, four of five in the mesalamine group, and one of two in the balsalazide group had final (week 8 or time of study termination) whole blood 6-TGN concentrations greater than the mean final whole blood 6-TGN concentration of $168 \mathrm{pmol} / 8 \times 10^{8} \mathrm{RBCs}$ for the entire study cohort. These findings suggest that elevated whole blood 6-TGN concentrations may be associated with the development of leucopenia in patients treated with AZA or 6-MP, and raise the possibility that therapeutic drug monitoring by measuring RBC or whole blood 6-TGN in patients with IBD might be useful. We have previously reported a lack of correlation between whole blood 6-TGN and both efficacy and toxicity in patients with IBD treated with AZA or 6-MP. ${ }^{11} 17$ One of those studies was a four month trial in which a fixed dose of AZA $(2.0 \mathrm{mg} / \mathrm{kg})$ was initiated in patients with active steroid treated $\mathrm{CD} .{ }^{17}$ Patients receiving mesalamine or sulphasalazine and those with low or intermediate TPMT activity were excluded from that study. We also performed a separate cross sectional study of a selected group of patients with IBD who had received AZA or 6-MP for a minimum of four months. ${ }^{11}$ The majority of the patients in this latter study were in symptomatic remission, some patients with known intermediate TPMT activity were receiving reduced doses of AZA or 6-MP, and patients with early dose dependent toxicity who had discontinued AZA or 6-MP were not included in the study. These factors may have led to the selection of relatively homogenous patient groups which would make demonstration of the utility of 6-TGN monitoring difficult. Nevertheless, in our cross sectional study we demonstrated a trend towards an increase in whole blood 6-TGN concentrations of patients receiving mesalamine, sulphasalazine, or olsalazine concomitantly with AZA or 6-MP. ${ }^{11}$ In the current study, the provocative manoeuvre of increasing whole blood 6-TGN concentrations, potentially through inhibition of TPMT with mesalamine or sulphasalazine, resulted in leucopenia in $50 \%$ of patients. This finding provides strong evidence that elevated whole blood 6-TGN concentrations may lead to leucopenia, as has been demonstrated repeatedly in studies of the use of thiopurine drugs to treat acute lymphoblastic leukaemia.

Other studies of 6-TGN therapeutic drug monitoring in patients with IBD, using an assay for RBC 6-TGN developed by Lennard, ${ }^{20}$ have reported that 6-TGN concentrations $>230$ $250 \mathrm{pmol} / 8 \times 10^{8} \mathrm{RBCs}$ are associated with an increased rate of efficacy, and that patients with leucopenia had a median 6-TGN concentration of $286 \mathrm{pmol} / 8 \times 10^{8} \mathrm{RBCs}^{21}{ }^{22}$ We used a different RBC 6-TGN assay developed by Erdmann ${ }^{13}$ and adapted it to whole blood. Therefore, our results are not directly comparable with those of previous studies. However, we have demonstrated in a blinded comparison a high degree of correlation between the two assays, with a conversion factor of approximately 1.6 to convert Erdmann assay results to Lennard assay results (unpublished data). Therefore, the mean final whole blood 6-TGN concentration of $168 \mathrm{pmol} / 8 \times 10^{8} \mathrm{RBCs}$ observed in our study would be comparable with a mean RBC 6-TGN concentration of 269 $\mathrm{pmol} / 8 \times 10^{8} \mathrm{RBCs}$ using the Lennard assay.

We have previously shown that compounds which contain 5-ASA cause in vitro inhibition of recombinant human TPMT with $\mathrm{IC}_{50}$ values for olsalazine, sulphasalazine, balsalazide, and mesalamine of $31 \mu \mathrm{M}, 104 \mu \mathrm{M}, 197 \mu \mathrm{M}$, and $1380 \mu \mathrm{M}$, respectively. ${ }^{8-10}$ Mean plasma concentrations of 5-ASA, N-acetyl 5-ASA, sulphasalazine, and balsalazide observed in our study are somewhat below these $\mathrm{IC}_{50}$ values. Nevertheless, given the increase in whole blood 
6-TGN (pharmacokinetic effect) that occurred in our study, and the occurrence of leucopenia (pharmacodynamic effect), it appears that these concentrations may be sufficient to inhibit TPMT activity in vivo. We believe that in vivo inhibition of TPMT by 5-ASA, $N$-acetyl-5ASA, and sulphasalazine is the likely mechanism that led to increased concentrations of whole blood 6-TGN and the development of leucopenia in our patients. Because duodenal mucosal 6-TGN concentrations did not increase during the eight week study, we believe that the in vivo site of TPMT inhibition by 5 -ASA and related compounds may be predominantly at other sites.

The case report detailing myelotoxicity resulting from a possible drug interaction between 6-MP and olsalazine occurred in a patient with TPMT activity at the lower end of the normal range. ${ }^{10}$ Six patients in our study had intermediate TPMT activity (two in each of the three treatment groups). In four of the six patients, the prestudy AZA or 6-MP dose was reduced by $25-50 \%$ or more, and leucopenia only occurred in one of these four patients during the study. The remaining two patients with intermediate TPMT activity were both receiving standard doses of AZA prior to the study, and both developed leucopenia during the study. These findings support the possibility that patients with intermediate TPMT enzyme activity may be at particular risk for developing leucopenia as a result of a drug interaction between AZA or 6-MP and suggest that the risk may be decreased by using a reduced dose of AZA or 6-MP, perhaps in conjunction with therapeutic drug monitoring with 6-TGN. Finally, there was some evidence of "induction" of TPMT activity in the mesalamine and balsalazide groups but the magnitude of the effect was not large and the pharmacological impact is likely limited.

Three of six patients in the sulphasalazine group who developed leucopenia did not have increased RBC 6-TGN concentrations. Interestingly, all three of these patients had total sulphapyridine plasma concentrations exceeding $50 \mu \mathrm{M}$, a threshold that has been associated with "slow acetylator" status and increased risk for sulphasalazine toxicity, including leucopenia and other forms of myelosuppression. ${ }^{23}$ Thus in patients treated with both AZA or 6-MP and sulphasalazine, slow acetylator status and elevated plasma concentrations of total sulphapyridine may be independent risk factors for the development of leucopenia.

It is possible that the drug interaction between AZA or 6-MP and mesalamine, sulphasalazine, or possibly balsalazide that we have reported here may actually benefit patients. As discussed previously, some investigators have reported that increased RBC concentrations of 6-TGN are associated with a greater likelihood of achieving remission in patients with CD treated with AZA or 6-MP. ${ }^{21}{ }^{22}$ Other investigators have suggested that the occurrence of mild leucopenia in patients with $C D$ treated with 6-MP is associated with a greater likelihood of achieving remission, and is therefore desirable. ${ }^{24}$ Thus it is reasonable to speculate that therapeutic synergy might occur in patients treated concomitantly with AZA or 6-MP and mesalamine, sulphasalazine, or balsalazide, mediated by increased whole blood 6-TGN concentrations and mild leucopenia. Conversely, patients in whom remission was induced by combination therapy with these agents might be expected to relapse if mesalamine, sulphasalazine, or balsalazide is withdrawn during the maintenance phase of therapy unless the dose of AZA or 6-MP is increased.

Oral mesalamine was well tolerated during this study. Even though mild leucopenia occurred in five patients $(50 \%)$, only one patient had to withdraw prematurely from the study for a WBC count $<3.0 \times 10^{9} / 1$. Sulphasalazine was only moderately well tolerated, with a high frequency of headaches and fatigue. Mild leucopenia occurred in six patients but only one withdrew from the study prematurely for a WBC count $<3.0 \times 10^{9} / 1$. Balsalazide was also only moderately well tolerated with a high frequency of worsening diarrhoea and nausea. Two patients developed mild leucopenia but did not require early withdrawal.

In summary, in patients with $\mathrm{CD}$ receiving AZA or 6-MP, coadministration of mesalamine or sulphasalazine and possibly balsalazide resulted in an increase in whole blood 6-TGN concentrations and a high frequency of mild leucopenia. This drug interaction probably results from inhibition of the thiopurine metabolising enzyme TPMT by benzoic acid derivatives such as mesalamine, sulphasalazine, and balsalazide. These pharmacokinetic and pharmacodynamic effects have the potential for both adverse and beneficial effects in patients. Health care providers should be aware of this drug interaction.

Supported by research grants from Procter \& Gamble, the Mayo Foundation, DK 07198-22, GM28157, and GM35720 from the NIH, and grant FD-T-000-886 from the FDA.

Conflicts of interest: Dr Sandborn is a consultant for Procter \& Gamble. Drs Sandborn, Loftus, and Tremaine participate in a continuing medical education program indirectly sponsored by Procter \& Gamble. Dr Weinshilboum and Ms Szumlanski have a licensing agreement with Variagenics, Cambridge, MA, for the commercialisation of molecular tests for thiopurine methyltransferase.

1 Pearson DC, May GR, Fick GH, et al. Azathioprine and 6-mercaptopurine in Crohn's disease: a meta-analysis. Ann Intern Med 1995;122:132-42.

2 Lennard L. The clinical pharmacology of 6-mercaptopurine. Eur f Pharmacol 1992;43:329-39.

3 Weinshilboum RM, Sladek SL. Mercaptopurine pharmacogenetics: monogenic inheritance of erythrocyte pharmacogenetics: monogenic inheritance of erythrocyte thiopurine

4 Szumlanski C, Otterness D, Her C, et al. Thiopurine methyltransferase pharmacogenetics: human gene cloning and characterization of a common polymorphism. DNA Cell Biology 1996;15:17-30

5 Otterness D, Szumlanski C, Lennard L, et al. Human thiopurine methyltransferase pharmacogenetics: gene sequence polymorphisms. Clin Pharmacol Ther 1997;62:6073.

6 Lennard L, Van Loon JA, Weinshilboum RM. Pharmacogenetics of acute azathioprine toxicity: relationship to thiopurine methyltransferase genetic polymorphism. Clin Pharmacol Ther 1989;46:149-54.

7 Black AJ, McLeod HL, Capell HA, et al. Thiopurine methyltransferase genotype predicts therapy-limiting severe tox-

8 Szumlanski CL, Weinshilboum RM. Sulphasalazine inhibition of thiopurine methyltransferase: possible mechanism for interaction with 6-mercaptopurine and azathioprine. $\mathrm{Br}$ f Clin Pharmacol 1995;39:456-9. 
9 Lowry PW, Szumlanski CL, Weinshilboum RM, et al. Balsalazide and azathioprine or 6-mercaptopurine: evi-
dence for a potentially serious drug interaction. Gastroenterdence for a potentially seric
ology 1999;116:1505-6.

10 Lewis LD, Benin A, Szumlanski CL, et al. Olsalazine and 6-mercaptopurine-related bone marrow suppression: a possible drug interaction. Clin Pharmacol Ther 1997;62 464-75.

11 Lowry PW, Franklin CL, Weaver AL, et al. Measurement of thiopurine methyltransferase activity and azathioprine metabolites in patients with inflammatory bowel disease. Gut 2001;49:665-70.

12 Irvine EJ, Feagan B, Rochon J, et al. Quality of life: a valid and reliable measure of therapeutic efficacy in the treatment of inflammatory bowel disease. Gastroenterology 1994;106:287-96.

13 Erdmann GR, France LA, Bostrom BC, et al. A reversed phase high performance liquid chromatography approach in determining total red blood cell concentrations of 6 thioguanine, 6-mercaptopurine, methythioguaine, and thioguanine, 6-mercaptopurine, methythioguaine, and methylmercaptopurine in a patient receivir

14 Bolieu R, Lenoir A. Determination of thiopurine nucleotides in human lung tissue by high-performance liquid chromatography. f Chromatogr B Biomed Appl 1995;665 213-16.

15 Awni WM, Braeckman RA, Locke CS, et al. The influence of multiple oral doses of zileuton on the steady-state pharmacokinetics of sulfasalzine and its metabolites, sulfapyridine and $\mathrm{N}$-acetylsulfapyridine. Clin Pharmacokinet 1995; 29(suppl 2):98-104.
16 Chan RP, Pope DJ, Gilbert AP, et al. Studies of two novel sulfasalazine analogs, ipsalazide and balsalazide. Dig Dis Sci sulfasalazine anal

17 Sandborn WJ, Tremaine WJ, Wolf DC, et al. Lack of effect of intravenous administration on time to respond to azathioprine for steroid-treated Crohn's disease. Gastroenterology 1999;117:527-35.

18 Connell WR, Kamm MA, Ritchie JK, et al. Bone marrow toxicity caused by azathioprine in inflammatory bowel disease: 27 years of experience. Gut 1993;34:1081-5.

19 Present DH, Meltzer SJ, Krumholz MP, et al. 6-Mercaptopurine in the management of inflammatory bowel disease: short- and long-term toxicity. Ann Intern Med 1989;111:641-9.

20 Lennard L. Assay of 6-thioinosinic acid and 6-thioguanine nucleotides, active metabolites of 6-mercaptopurine, in human red blood cells. 7 Chromatogr 1987;423:169-78.

21 Dubinsky MC, Lamothe S, Yang $\mathrm{H}$, et al. Pharmacogenomics and metabolite measurement for 6-mercaptopurine therapy in inflammatory bowel disease. Gastroenterology 2000;118:705-13.

22 Cuffari C, Theoret Y, Latour S, et al. 6-Mercaptopurine metabolism in Crohn's disease: correlation with efficacy

23 Das KM, Eastwood MA, McManus JPA, et al. Adverse reactions during salicylazosulfapyridine therapy and the relation with drug metabolism and acetylator phenotype. $N$ Engl F Med 1973;289:491-5.

24 Colonna T, Korelitz BI. The role of leukopenia in the 6-mercaptopurine-induced remission of Crohn's disease. Am $\mathcal{F}$ Gastroenterol 1994;89:362-6.

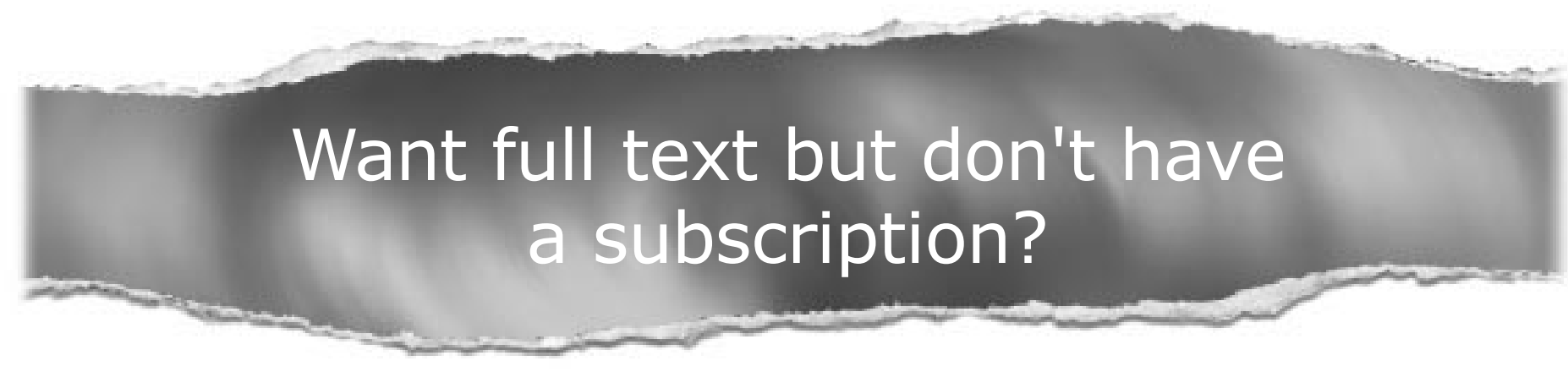

\section{Pay per view}

For just \$8 you can purchase the full text of individual articles using our secure online ordering service. You will have access to the full text of the relevant article for 48 hours during which time you may download and print the pdf file for personal use.

www.gutjnl.com 